\title{
Actions de vulgarisation du guichet national de formation continue du GIP-CNFM
}

\author{
B. Pradarelli ${ }^{\mathrm{a}}$, P. Nouet ${ }^{\mathrm{a}}$, P. Benoit ${ }^{\mathrm{a}}$, O. Bonnaud ${ }^{\mathrm{b}}$ \\ ${ }^{a}$ LIRMM et pôle CNFM de Montpellier (PCM), Université de Montpellier, France \\ ${ }^{\mathrm{b}}$ IETR (Université de Rennes 1) et GIP-CNFM, Grenoble, France \\ Contact email : beatrice.pradarelli@umontpellier.fr
}

Cet article présente les actions de dissémination et de vulgarisation mises en œuvre pour promouvoir l'offre de formation continue en Microélectronique et en Nanotechnologies du réseau GIP-CNFM. Il rappelle la structuration et organisation du guichet d'entrée national et dresse le bilan 2014-2018 des formations continues réalisées dans le cadre du projet ANR IDEFI-FINMINA. Il conclut en adressant l'actualité et les nouvelles actions identifiées pour accroître la visibilité du GIPCNFM dans le milieu de la formation professionnelle continue.

Mots clefs : formation continue, microélectronique, nanotechnologies, reconnaissance, visibilité.

\section{Introduction}

L'évolution de la microélectronique depuis les années 60-70 a contribué à modifier le paysage socio-économique de notre pays dans les domaines de l'informatique, de la communication, des transports, de l'environnement, de l'énergie et de la médecine. Elle a permis à la fois l'augmentation de la productivité dans tous les secteurs d'activité et le passage d'une industrie lourde en décroissance vers des activités innovantes à forte valeur ajoutée. Cette mutation a entrainé la nécessité de disposer de plateformes technologiques dédiées et d'un support technique associé pour permettre l'enseignement des savoirs et des savoir-faire en microélectronique et nanotechnologies.

Afin de répondre à ces besoins de formation, et compte tenu de l'importance des investissements à consentir, un réseau de 12 pôles interuniversitaires ( $c f$. Figure 1) a été créé il y a près de 40 ans, regroupant et mutualisant des moyens opérationnels tout en définissant une répartition géographique adaptée à la présence d'industries, de laboratoires de recherche et d'établissements de formation.

Ces pôles sont fédérés depuis 2002 par le Groupement d'Intérêt Public pour la Coordination Nationale de la Formation en Microélectronique et en nanotechnologies (GIP CNFM).

Depuis sa création, une des missions du GIP-CNFM $(1,2)$ consiste à mutualiser des moyens opérationnels (matériels et logiciels) qu'un établissement seul aurait du mal à « rentabiliser », afin de les mettre à disposition des enseignants-chercheurs, chercheurs et ingénieurs des universités, écoles d'ingénieurs et laboratoires de recherche de France dans le but de former efficacement les étudiants des filières micro et nanoélectronique.

Une autre mission consiste à former, de façon permanente et tout au long de leur vie, les enseignants-chercheurs, ingénieurs, chercheurs et doctorants afin qu'ils maitrisent des technologies aussi indispensables qu'en constante évolution.

Lauréat de l'appel à projet Initiative d'Excellence pour les Formations Innovantes avec le projet IDEFI-FIMINA (2012-2019) (3-5), le GIP-CNFM a mis en place un 
guichet national d'entrée pour la formation continue et tout au long de la vie à destination des acteurs des secteurs privé et public (6).

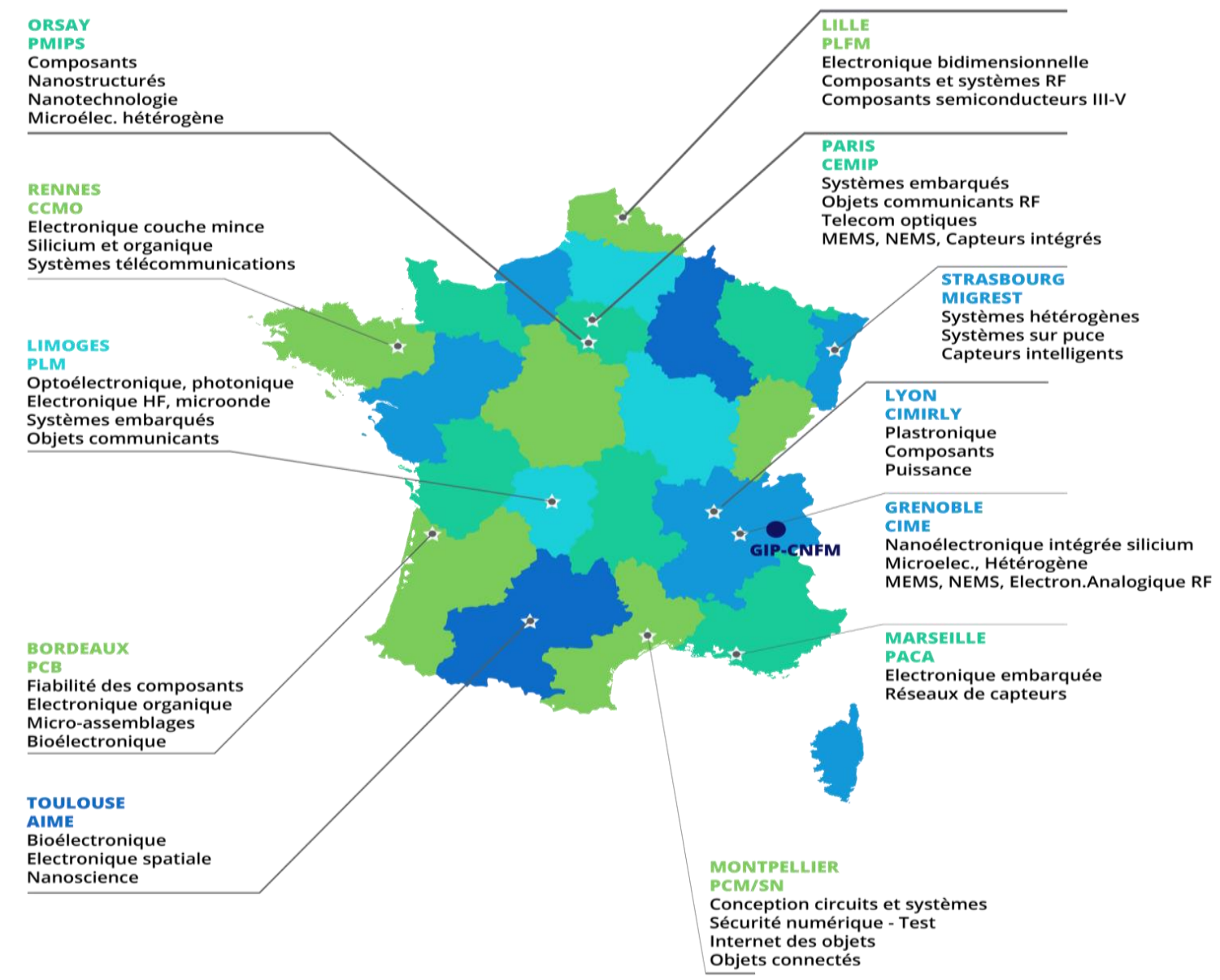

Fig.1. Réseau des 12 pôles du GIP-CNFM. Ils mutualisent les moyens opérationnels qu'ils soient technologiques ou de conception avec des plateformes dédiées et des Services Nationaux accessibles à toutes les formations académiques.

Cet article est organisé en cinq parties. Suite à cette introduction, la seconde partie présente l'activité de formation continue du GIP-CNFM pour les publics académique et industriel, en rappelant la structuration du guichet national d'entrée, l'organisation du site internet des Services Nationaux qui est le site vitrine de l'offre de formation continue nationale et les moyens qui sont mis en œuvre pour la promouvoir. La troisième partie est dédiée au bilan 2014-2018 de l'action de formation continue pour les personnels académique et industriel. La quatrième partie informe sur l'actualité récente en lien avec le bilan. La dernière conclut et adresse les perspectives d'amélioration de la reconnaissance du GIP-CNFM en tant qu'acteur reconnu de la formation professionnelle continue.

\section{Activité de formation continue du GIP-CNFM}

L'activité de formation continue comprend deux volets :

- Des actions de formation dédiées uniquement aux académiques (enseignantschercheurs, doctorants, chercheurs, ingénieurs de recherche). Les formations proposées sont dispensées par les formateurs de nos partenaires industriels des logiciels de conception de circuits intégrés (Cadence, Silvaco, Mentor Graphics, 
Coventor, Synopsys) ou des plateformes de prototypage (Xilinx, Intel, Digilent). Ces formations sont organisées par les Services Nationaux du GIP-CNFM.

- Des actions de formations pour les publics académique et industriel, dispensées par des formateurs académiques, sur l'ensemble des moyens opérationnels du réseau GIP-CNFM dont les centrales technologiques de fabrication et les plateformes technologiques de test et de sécurité numérique.

Cette organisation est résumée par la Figure 2.

\begin{tabular}{|c|c|c|c|c|c|c|c|c|}
\hline Offre & \multicolumn{2}{|c|}{$\begin{array}{l}\text { Public } \\
\text { Aca Ind }\end{array}$} & \multicolumn{2}{|c|}{ Formateurs } & \multicolumn{3}{|c|}{ Formations } & Planification \\
\hline $\begin{array}{l}\text { Services } \\
\text { Nationaux }\end{array}$ & Q & 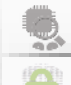 & Q & П & $>4$ & Q & П & Annuelle \\
\hline 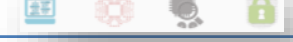 & & & & & & & & \\
\hline $\begin{array}{l}\text { Réseau } \\
\text { GIP-CNFM }\end{array}$ & Q & Q & प & & 64 & Q & & Annuelle \\
\hline
\end{tabular}

Fig.2. Organisation de l'offre de formation continue. Les plateformes peuvent être celles des Services Nationaux du GIP-CNFM, ou bien celles des différents pôles répartis sur le territoire.

Offre de formation continue des Services Nationaux

Les Services Nationaux du CNFM (7) ont été créés en 1986 pour permettre l'achat mutualisé de licences de CAO pour la conception de composants et systèmes microélectroniques. En 1996, une initiative similaire est lancée pour l'acquisition et la mutualisation d'un équipement technologique national pour le Test Industriel de circuits intégrés microélectroniques. L'équipement a été renouvelé en 2006 et est toujours utilisé pour les formations initiales et continues à Montpellier ou depuis des sites distants.

Une troisième plateforme consacrée à l'achat et à la diffusion de circuits et de cartes programmables sera lancée plus tard avant d'être complétée par deux plateformes, consacrées à la Formation Continue et à la Sécurité Numérique du matériel, mises en place dans le cadre de l'Initiative D'Excellence pour les Formations Innovantes (IDEFI) en 2012 (3).

Dédiés à l'enseignement en formation initiale et à la recherche, l'évolution permanente des outils logiciels et matériels proposés par les 5 plateformes nécessite que les utilisateurs académiques (enseignants-chercheurs, chercheurs, doctorants, ingénieurs de recherche) se forment régulièrement tout au long de leur carrière afin de maîtriser ces technologies.

Chaque année, les Services Nationaux établissent un calendrier de formations en collaboration avec les responsables des services formation des sociétés fournissant les suites logicielles et matérielles pour les plateformes Conception et Prototypage, et avec les enseignants-chercheurs de l'Université de Montpellier pour les plateformes Sécurité Numérique et Test Industriel.

Ce calendrier est ensuite diffusé à l'ensemble des utilisateurs des établissements d'enseignement supérieur de l'hexagone. L'offre concerne :

- Formations aux outils de conception de circuits intégrés et systèmes électroniques (Cadence, Synopsys, Mentor Graphics) $(8,9,10)$,

- Formations aux outils de prototypage (INTEL, Xilinx, DIGILENT) $(11,12,13)$,

- Formations à la sécurité numérique (14),

- Formations au test industriel (15), 
- Formations en e-learning proposé par la société Cadence pour la conception de circuits intégrés : ILS (8).

Les formations sont organisées soit à l'Université de Montpellier où les Services Nationaux sont hébergés, soit dans l'un des pôles du CNFM, soit dans une école du réseau, comme Télécom ParisTech (16). Depuis 2018, le pôle CNFM de Montpellier est reconnu comme une plateforme technologique de l'Université de Montpellier (17).

\section{Offre de formation continue du réseau GIP-CNFM}

Cette offre résulte de la consultation des enseignants-chercheurs des 12 pôles du réseau CNFM afin de recenser les compétences en micro-électronique et nanotechnologies et les moyens opérationnels mutualisés. Ces derniers sont notamment des suites logicielles pour le design et le prototypage de circuits intégrés, des centrales technologiques pour la fabrication et la caractérisation de composants et un testeur industriel pour la vérification de circuits. Le réseau CNFM dispose, de plus, de salles de cours dans tous les établissements d'enseignement supérieur du réseau.

Le but est de répondre aux besoins de l'industrie, dont le principal syndicat professionnel du domaine est représenté à la Présidence du GIP, en liaison avec les services de formation continue des établissements d'enseignements (Universités et écoles d'ingénieurs) afin d'améliorer la compétitivité des entreprises de ce secteur économique, de les aider à porter les innovations technologiques, de réactualiser les compétences des employés ou d'en développer de nouvelles pour améliorer leur employabilité et permettre la réalisation de projets de valorisation de carrières.

C'est une offre consolidée qui propose des formations continues allant de la sensibilisation à la spécialisation, alliant l'excellence des enseignements théoriques et la pratique sur du matériel de dernière génération pour l'acquisition de savoirs et savoirfaire, qu'il s'agisse de remise à niveau de fondamentaux ou de l'apprentissage de compétences avancées.

Cette offre dématérialisée se présente sous la forme d'un catalogue de fiches de formation harmonisées, chacune provenant d'un pôle CNFM. Chaque fiche renseigne sur la thématique de la formation en précisant les objectifs et les acquis de l'apprentissage, les prérequis, le programme journalier, la durée et les horaires, le lieu, les contacts pour l'inscription et les renseignements pédagogiques, les conditions de validation des acquis, le coût par participant et leur nombre minimum pour l'organisation de la formation. Ces dernières informations permettent de garantir la rentabilité de chaque formation car elles intègrent les coûts complets, c'est-à-dire les frais internes (salle de formation, supports de cours, utilisation des moyens opérationnels du GIP-CNFM pour les travaux pratiques, rémunération du formateur, ...) et ceux externes (gestion de la formation par le service de formation continue).

Tout personnel académique peut proposer une formation continue et ainsi enrichir la base de données, nommée GECO (18). Cette base de données supporte trois plateformes hébergées par les Services Nationaux : CAO, FPGA et FORMATION (cf. Figure 3).

Depuis la plateforme FORMATION, on peut consulter le catalogue, se connecter pour proposer une formation ou en modifier une existante. Pour créer une nouvelle fiche de formation, il est nécessaire de posséder un compte utilisateur en effectuant une requête auprès de l'administrateur.

Les formations sont classées par pôle CNFM (cf. Figure 4). La sélection de l'option « Proposer une formation » affiche le modèle de fiche que l'utilisateur va remplir. 


\section{Bienvenue sur les plateformes des services nationaux}

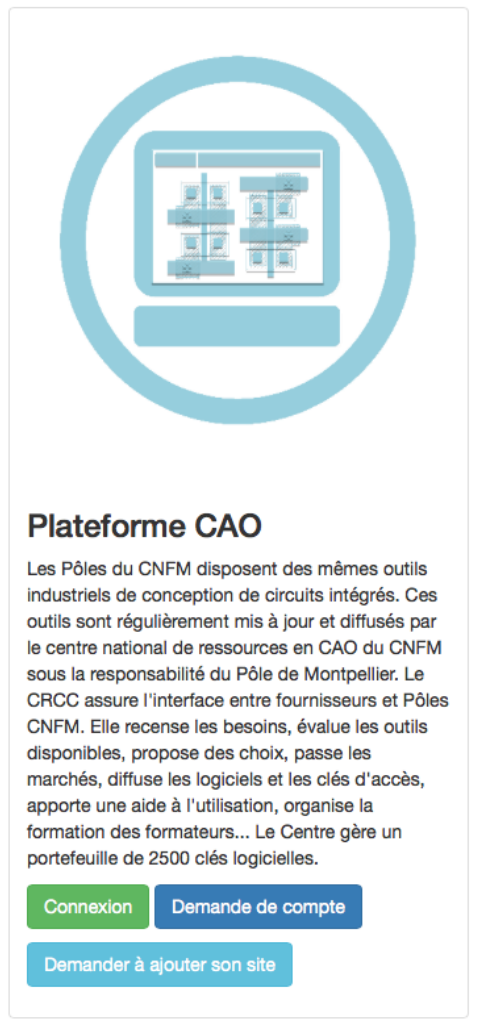

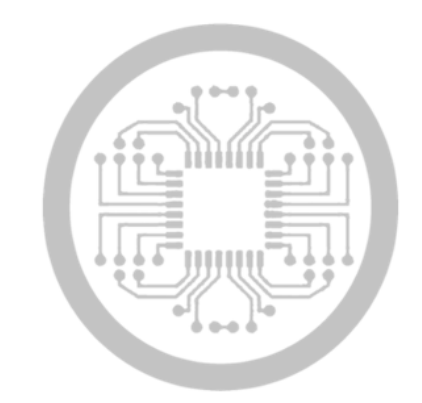

Plateforme FPGA

Pour tout renseignement, demande de devis ou commande, contactez fpga@enfm.fr

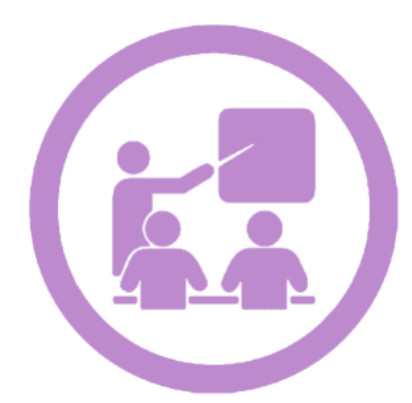

\section{Plateforme Formation}

Plateforme permettant d'accéder aux formations. Vous aurez ainsi la possibilité de vous inscrire, proposer une formation, etc.

\section{Connexion Demande de compte} Consulter le catalogue

Fig.3. Page d'accueil de la base de données GECO offrant les trois types d'activité de formation continue : la CAO (conception assistée par ordinateur) sur des outils dédiés et utilisés par l'industrie, le prototypage sur circuits programmable (FPGA) s'inscrivant dans le concept de l'électronique embarquée, ainsi que les autres formations qui peuvent être sélectionnées dans un catalogue ou à la carte.

Cette nouvelle formation sera ensuite validée par l'administrateur de la plateforme FORMATION et publiée sur le site internet de la formation continue du CNFM (19). Le catalogue comptabilise actuellement 64 fiches de formations continues (FC) courtes dont voici le détail :

- MIGREST, Pôle CNFM de Strasbourg, Nancy et Metz : 11 FC courtes,

- PACA, Pôle CNFM de Marseille et Nice : 25 FC courtes (18),

- PCM, Pôle CNFM de Montpellier : 8 FC courtes (6),

- PFLM, Pôle CNFM de Lille : 7 FC courtes (21),

- CIMIRLY, Pôle CNFM de Lyon : 4 FC courtes,

- PMIPS, Pôle CNFM de Paris Sud : 8 FC courtes,

- AIME, Pôle CNFM de Toulouse : 1 FC courte. 


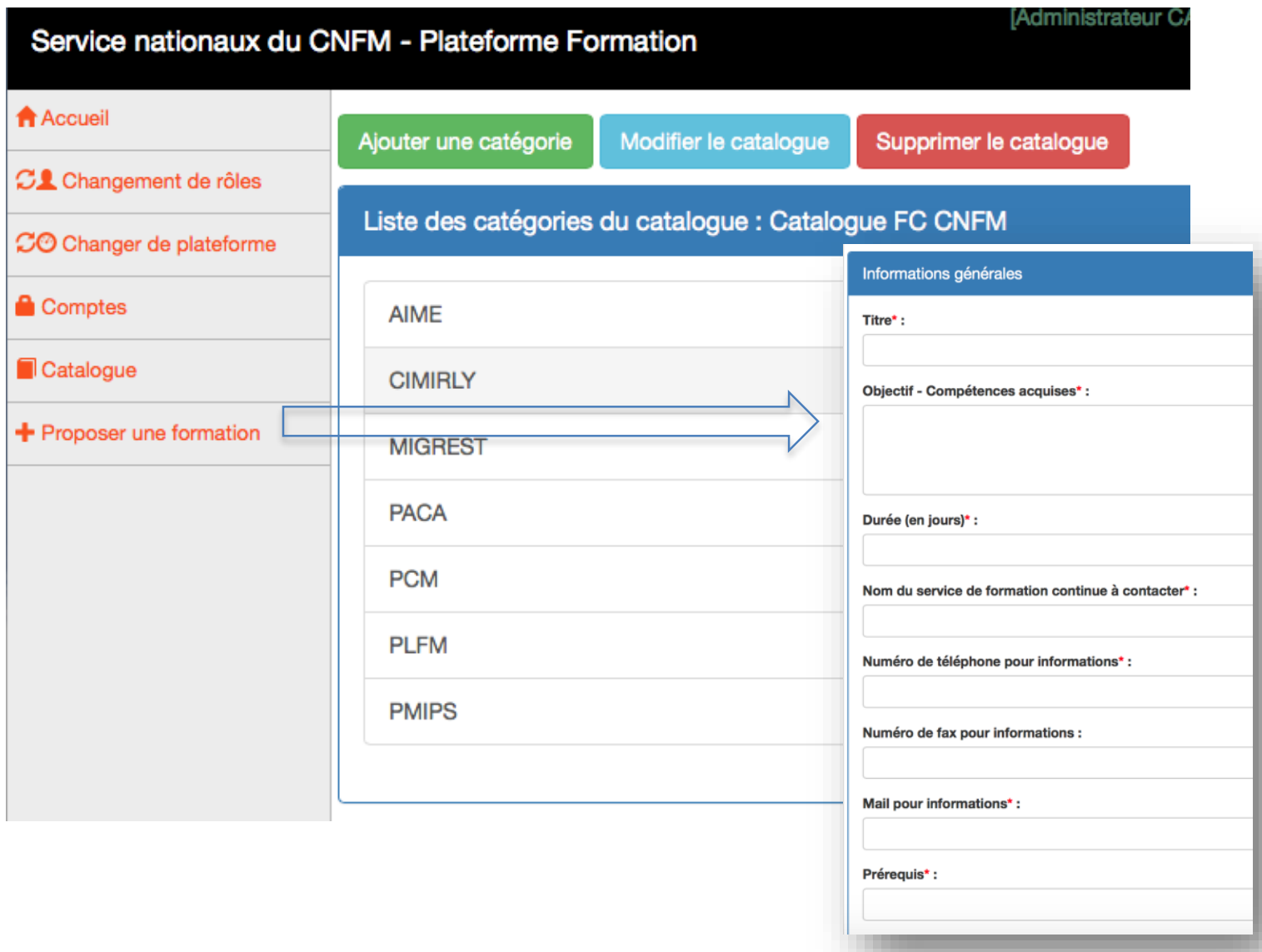

Fig.4. Processus de création d'une fiche de formation. Cette dernière peut être sélectionnée dans le catalogue ou être bâtie à la carte. Les demandeurs doivent demander au préalable une inscription auprès de l'administrateur du site.

\section{Guichet d'entrée national de formation continue du GIP-CNFM}

Lorsqu'une personne consulte en ligne le catalogue de formation, elle navigue de façon transparente entre le site internet (19) et la base de données GECO (18) de l'offre de formation du guichet national du GIP-CNFM.

Le GIP-CNFM s'appuie sur les SFC (Services de Formation Continue) des Universités et des Ecoles d'ingénieurs auxquels chaque pôle du réseau est rattaché, pour la gestion des demandes de formations émanant des entreprises et/ou des employés ainsi que pour l'organisation des formations. Ces structures interfacent avec les OPCA (Organismes Paritaires Collecteurs Agréés) pour les demandes de prise en charge des formations dans le cadre du CPF (Compte Personnel de Formation). Ils font aussi la promotion des formations au sein des bassins d'activités locaux une fois la maquette financière de chaque formation validée par le CEVU (Conseil des Etudes et de la Vie Etudiante).

Des réunions, ayant pour objectif le rapprochement des acteurs de la formation continue, organisées entre juillet et novembre 2015, ont permis d'établir des collaborations avec les services de formation continue des Universités de Strasbourg (20), d'Aix-Marseille (23), Montpellier (24), de Lille (25), de Paris Sud (26) et de Nice (27), de l'INSA Lyon (INSAVALOR, 28), de l'INSA Toulouse (29) et de Grenoble INP (30). 
Des conventions ont été signées avec les SFC des Universités de Strasbourg, d'AixMarseille, de Lille et de Montpellier pour la diffusion de l'offre GIP-CNFM sur leurs sites internet et l'organisation de formations.

Ces conventions placent ainsi les collaborations avec les SFC dans le cadre légal de la formation professionnelle continue, garantissant l'application des dernières lois sur le $\mathrm{CPF}$ et permettant la délivrance de certificats de participation.

\section{$\underline{\text { Diffusion }}$}

L'offre de formation continue est aussi diffusée par le GIP-CNFM à travers ses moyens de communication et lors de la participation à des évènements régionaux et nationaux, à savoir :

- Moyens de communication du GIP-CNFM :

- Site internet de la plateforme de formation continue du GIP-CNFM (19) : toutes les annonces de formations sont publiées dans la rubrique «Actualité » et leur programmation se retrouve sur le «Calendrier »,

- Toute annonce faite depuis le site internet est automatiquement relayée sur les différents réseaux sociaux à savoir Facebook (31), LinkedIn (32) et Twitter (33). Des articles spécifiques sont aussi postés sur ces médias de communication.

- Evènements (salons, projets) :

○ Participation du GIP-CNFM à l'élaboration du Livre Blanc qui établit la feuille de route de la filière Electronique Française pour les dix ans à venir et qui a été présenté dans le cadre du WEF (World Electronic Forum) qui s'est tenu à Angers fin octobre 2017,

- Conférence du GIP-CNFM au salon ENOVA de Toulouse fin mai 2018

- Conférences du GIP-CNFM à la JRE (Journée Régionale de 1'Electronique) d'ACSIEL (34), notre partenaire industriel privilégié, en décembre 2017 à Lille, en avril 2018 à Caen et en octobre 2018 à Grenoble,

- Participation du GIP-CNFM à la Commission Affaires Sociales - DRH d'ACSIEL en mars, juin et septembre 2018, et janvier 2019.

\section{Bilan de la formation continue 2014-2018}

Le tableau I présente le bilan de la formation continue, tous publics confondus, sur la période 2014 à 2018. Par année, le nombre de jours de formation et le nombre de personnes formées en présentiel sont confrontés aux indicateurs du projet IDEFIFINMINA.

Ces indicateurs ont été établis en prévoyant une progression annuelle constante. Ce bilan montre certes une croissance mais les résultats de la formation continue présentielle ne sont pas à la hauteur des objectifs même s'ils s'en rapprochent.

TABLEAU I. Bilan de la formation continue «présentielle » du GIP-CNFM dans le cadre du projet IDEFI-FINMINA

\begin{tabular}{ccc}
\hline Année & Jours / indicateurs & H*pers / indicateurs \\
\hline 2014 & $30 / 36$ & $2366 / 2500$ \\
2015 & $37 / 40$ & $2089 / 2750$ \\
2016 & $15 / 45$ & $994 / 3000$ \\
2017 & $24 / 50$ & $2199 / 3400$ \\
2018 & $33 / 55$ & $2887 / 3600$ \\
2019 & $-/ 60$ & $-/ 4000$ \\
\hline
\end{tabular}


$\mathrm{Si}$, par contre, on rajoute les résultats de formation continue en-ligne, provenant essentiellement de l'offre Cadence mise en place en 2016, alors les indicateurs sont globalement atteints en 2017 et 2018.

TABLEAU II. Bilan de la formation continue «présentielle et en ligne» du GIP-CNFM dans le cadre du projet IDEFI-FINMINA

\begin{tabular}{ccc}
\hline Année & Jours / indicateurs & H*pers / indicateurs \\
\hline 2014 & $30 / 36$ & $2366 / 2500$ \\
2015 & $37 / 40$ & $2089 / 2750$ \\
2016 & $15 / 45$ & $994 / 3000$ \\
2017 & $110 / 50$ & $2801 / 3400$ \\
2018 & $652,5 / 55$ & $7223,5 / 3600$ \\
2019 & $-/ 60$ & $-/ 4000$ \\
\hline
\end{tabular}

L'analyse détaillée des données de formation continue en fonction du public ciblé a permis d'identifier certaines raisons pouvant expliquer la croissance un peu faible.

\section{Bilan 2014-2018 de l'offre de formation continue des Services Nationaux}

Des 5 partenaires industriels fournisseurs de logiciels de CAO des Services Nationaux, Cadence, Synopsys, Coventor, Silvaco et Mentor Graphics, seul Cadence a développé depuis le début une politique de formation continue pour le public académique. Ainsi, chaque année, une ou plusieurs formations ont été organisées et délivrées par un formateur de la société sur des sites CNFM ou dans les locaux de Cadence en région parisienne.

Lors du dernier contrat quadriennal signé avec Cadence en 2014-2018, l'offre de formation continue jusque-là uniquement présentielle s'est vue élargie à l'apprentissage en ligne. La gratuité de cette offre pour les utilisateurs combinée à la flexibilité des horaires de formation et à la réduction des coûts de déplacement ont été les facteurs majeurs de la montée en puissance de cette nouvelle forme de formation au détriment du présentiel.

TABLEAU III. Evolution de demande de formation continue Cadence de 2014 à 2018 dans le cadre du projet IDEFI-FINMINA du GIP-CNFM : émergence de l'apprentissage en e-learning par rapport au présentiel

$\begin{array}{ccc}\text { Année } & \text { Participants en présentiel/e-learning } & \text { H*pers présentiel/e-elearning } \\ 2014 & 8 / 0 & 168 / 0 \\ 2015 & 20 / 0 & 486 / 0 \\ 2016 & 8 / 0 & 154 / 0 \\ 2017 & 0 / 4 & 0 / 602 \\ 2018 & 17 / 21 & 329 / 4336,5\end{array}$

Avec le e-learning, un même utilisateur suit plus de formations, ce qui explique la différence de volume horaire. Pour chaque formation proposée en ligne, Cadence indique le nombre de jours équivalent en présentiel. Ces volumes horaires mentionnés ci-dessus ont été calculés en supposant qu'une journée de formation en présentiel dure $7 \mathrm{~h}$.

Rejoignant les fournisseurs d'outils de conception de circuits intégrés en 2017, Mentor Graphics propose aussi un ensemble de formations continues pour le personnel académique. A ce jour, une seule formation a été organisée.

Les formations aux outils de CAO sont donc en décroissance mais cette tendance est largement compensée par les formations sur les plateformes de prototypage proposées par 
Xilinx, Digilent et Intel. Ces dernières années, on constate un véritable engouement pour les cartes FPGA (Field Programmable Gate Array) de la part des enseignants chercheurs. En effet, les fonctionnalités accrues qu'offrent les dernières cartes leur permettent de proposer aux étudiants des filières électroniques des projets innovants dans lesquels ils peuvent aborder l'aspect système du composant électronique. L'évolution technologique rapide des plateformes de prototypage nécessite la formation régulière du personnel académique. Ces dernières années, Xilinx et Digilent ont mis en place une politique de séminaires et de formations, à la journée, avec une gratuité totale pour le public académique.

En 2017-2018, cette activité de formation représente 762 h.personnes pour 82 utilisateurs formés.

Le test industriel étant une activité du domaine des semi-conducteurs peu représentée sur le territoire Français, la demande de formation continue sur la plateforme technologique des Services Nationaux est peu élevée mais constante.

Enfin, l'analyse des chiffres relatifs aux actions de formation continue sur la plateforme de sécurité numérique montre que cette thématique est en plein essor et la demande en pleine croissance. Cependant, contrairement aux plateformes de CAO et de prototypage qui bénéficient de formateurs externes, les formations continues proposées sur les plateformes de test industriel et de sécurité numérique sont réalisées par quelques enseignants-chercheurs seulement, essentiellement de l'Université de Montpellier, qui ont des disponibilités réduites. D'autre part, les formations continues relatives à la sécurité des systèmes électroniques ne sont qu'au début de leur développement et il est pertinent de penser que leur croissance va aller de pair avec l'augmentation de l'utilisation des objets connectés.

\section{Bilan de l'offre de formation continue du réseau GIP-CNFM}

La formation continue auprès du public industriel n'a pas reçu l'accueil attendu et on ne constate pas de croissance significative. Diverses raisons expliquent ce bilan :

- Le GIP-CNFM manque de visibilité et de reconnaissance comme fournisseur de solution de formations continues en microélectronique et nanotechnologie malgré les actions de communication,

- Les formations continues proposées adressent des thématiques pointues, ciblant souvent un public de niveau ingénieur et sont non diplômantes,

- La difficulté de synchroniser la disponibilité des formateurs (enseignantschercheurs) avec celle des industriels réduit l'organisation de formations,

- Les contextes économiques et légaux ont énormément évolué depuis le début du projet IDEFI-FINMINA avec notamment une réglementation plus stricte de l'accès à la formation professionnelle continue, ce qui a pour conséquence une réduction des demandes,

- Enfin le catalogue en ligne est un bel outil permettant de montrer la diversité des compétences que les acteurs du secteur privé à la recherche d'une formation peuvent acquérir en venant se former au CNFM. Cependant, toutes les formations continues qui ont été réalisées, sont des formations sur mesure organisées pour une société et un petit nombre de ses personnels, ce qui n'a pas permis de capitalisation, à l'exception du plan de formation développé par le pôle CNFM PACA de Marseille pour la société ST Microelectronics, site de Rousset (35). 
Pour finir sur une note positive et optimiste, il ressort de l'évaluation des formations réalisées, une très grande satisfaction des apprenants qui ont apprécié la pédagogie des formateurs et les moyens opérationnels mis à leur disposition.

\section{Actualités du réseau CNFM « Formation Continue »}

Afin d'accroître la visibilité du GIP-CNFM en tant que fournisseur de solution de formations continues et d'élargir les thématiques proposées par les formations du catalogue, une série d'actions ont été menées en 2018 :

- La réalisation d'un clip vidéo présentant le GIP-CNFM et ses actions pour la formation continue (https://www.youtube.com/watch?v=IiObx9KuhBQ). Cette production résulte de la collaboration entre le pôle CNFM de Montpellier et la société montpelliéraine Artcam Production (36) spécialisée dans l'élaboration de films en motion-design. Ce clip, très dynamique, présente, par l'intermédiaire d'une voix off (voir le texte en Figure 5), l'organisation du réseau CNFM, les moyens opérationnels, les thématiques portées par les pôles ainsi que les collaborations avec les différents SFC pour la réalisation de formations professionnelles continues. Ce clip a été présenté lors des dernières JPCNFM (Journées Pédagogiques du CNFM) à Saint Malo début novembre 2018. Il a reçu un accueil très chaleureux de la part de tous les participants,

\footnotetext{
Vous êtes employé, employeur ou demandeur d'emploi à la recherche de formations continues dans les domaines de l'électronique, de la microélectronique et des systèmes embarqués ? Vous souhaitez acquérir de nouvelles compétences ou renforcer votre expertise professionnelle?

Les formations proposées par le GIP CNFM sont faites pour vous! Qu'est-ce que le GIP CNFM?

La Coordination Nationale pour la Formation en Microélectronique et Nanotechnologies est un Groupement d'intérêt Public. Sa mission principale est de mettre des moyens opérationnels mutualisés à la disposition de tous les établissements de l'enseignement supérieur et des laboratoires de recherche pour favoriser l'acquisition de compétences et l'innovation technologique.

Le GIP-CNFM est un réseau de 12 pôles, chacun regroupant différentes spécialités au sein d'universités et d'écoles d'ingénieurs. Huit pôles (Grenoble, Toulouse, Bordeaux, Limoges, Rennes, Paris-Sud, Paris et Lille en visuel) disposent de salles blanches et de centrales technologiques pour la fabrication et la caractérisation de nouveaux composants nano/microlélectroniques intelligents dédiés aux domaines de l'industrie, des transports, de la santé, de la vie domestique et bien d'autres encore.

Les autres pôles (Montpellier, Nice \& Marseille, Strasbourg \& Metz \& Nancy et Lyon en visuel) sont spécialisés dans la conception de circuits intégrés, de systèmes embarqués et d'objets connectés, (en plastronique, en électronique spatiale et biologique : coupé dans la version diffusée).

Intégrant les besoins métiers manifestés par son partenaire industriel ACSIEL, l'alliance des professionnels Français alimentant la chaîne de valeur de l'Electronique, GIP CNFM propose un catalogue de formations continues courtes orientées compétences. Vous serez immédiatement opérationnel grâce aux savoirs et savoir-faire que vous aurez acquis.

Les formations existantes ne correspondent pas à votre besoin? Venez nous en parler et élaborons ensemble le programme technique et pédagogique sur mesure qui y répondra. Nous déterminerons aussi les dates et le lieu de formation les plus adéquats.

Car le réseau CNFM e'est aussi un tissage national d'espaces d'apprentissage dans lesquels vous aurez plaisir à étudier, en bénéficiant de l'écoute attentive de nos formateurs.

Vous avez des heures dans votre CPF ? Les services de formation continue des universités pourront vous aider dans la mise en auvre de la prise en charge de votre formation continue et de sa validation.

Venez vivre l'expérience de vous former au CNFM où pédagogie innovante, expertise technique et moyens opérationnels sont réunis pour vous offrir les meilleures conditions pour améliorer votre employabilité !

Si vous souhaitez en savoir plus, rendez-vous sur web-pcm.cnfm.fr pour découvrir notre catalogue de formations continues ou contactez-nous à : formation-continue@cnfm.fr
}

Fig.5. Texte de la voix off du clip en motion-design. Il contient les informations pertinentes de présentation du réseau, de son organisation, de ses ressources et compétences, ainsi que de ses possibilités de formations. 
- La création de la chaîne YouTube «CNFM COMMUNICATON » (37) pour le lancement du clip. Le clip a aussi été relayé sur le site des services nationaux et ses comptes sur les 3 réseaux sociaux, FB, LinkedIn et Twitter,

- Le lancement de la première formation diplômante du réseau GIP-CNFM par le pôle CNFM PACA Marseille en collaboration avec l'école Polytech Marseille et le SFC de l'AMU (Aix-Marseille Université). Il s'agit du DESU (Diplôme d'Etudes Supérieures Universitaires) ingénieur produit en microélectronique. La formation se déroule sur 32,5 jours à raison de 2 jours par semaine et comprend 9 modules d'enseignement (38). Les modules sont capitalisables sur une durée maximale de 2 ans. Ce pôle a également enrichi son offre locale de formations continues professionnelles en adressant de nouvelles thématiques à savoir l'électronique RF (Radio Fréquence) (39), les protocoles RF (40), les techniques de fiabilité (41), les méthodes statistiques et bases de données (42),

- Le lancement de formations professionnelles continues sur la programmation de microcontrôleur (43) par 1'IUT GEII de Marseille, le pôle CNFM PACA Marseille et le SFC de l'AMU (Aix-Marseille Université).

\section{Conclusion et perspectives}

Le bilan des 4 dernières années fait apparaître des changements profonds quant au positionnement des apprenants des secteurs public et privé face à la nécessité de se former régulièrement. L'apprentissage en ligne a souvent supplanté la formation en présentiel pour des raisons économiques et de disponibilité. De plus, les 2 réformes de la formation professionnelle continue ont contribué à réglementer de façon plus stricte l'accès à la formation.

Grâce à ses atouts pédagogiques et opérationnels ainsi qu'à des efforts très importants en matière de communication, le réseau GIP-CNFM est maintenant identifié comme un acteur central de la formation continue dans le domaine de la microélectronique. Il n'en reste pas moins que le nombre d'heures de formation ou de personnes formées, en présentiel, est inférieur à nos attentes.

Cette situation devrait évoluer dans le cadre de la mise en place de la Filière Electronique par la Direction générale des Entreprises (DGE) au cours de l'année 2019. Des groupes de travail dans le cadre du Comité de suivi de filière (CSF) dans lequel sont impliqués des animateurs du réseau CNFM devraient pouvoir dégager de façon plus précise les besoins des entreprises (nature et niveau de formation) et leurs préférences dans l'organisation des sessions ou cycles de formation (groupes disciplinaires ou pluridisciplinaires, localisation, durée, fragmentation, validation et qualification).

Les objectifs du guichet national pour la formation continue du GIP-CNFM visent principalement trois axes d'amélioration :

- Reconnaissance et visibilité du réseau GIP-CNFM : améliorer le référencement de notre site internet en liaison avec nos partenaires, réaliser des séminaires et des formations courtes en synergie avec d'autres acteurs comme CAPTRONIC, utiliser encore plus les médias numériques tels que la chaîne YouTube et les réseaux sociaux comme support promotionnel de l'offre de formation continue,

- Élargissement du positionnement vers les objets communicants, les systèmes embarqués et l'électronique en général afin de couvrir les besoins des entreprises au-delà du seul domaine de la microélectronique, 
- Diversification de l'offre de formation continue pour qu'elle soit accessible à un niveau technicien en plus de celui d'ingénieur.

Un ensemble de formations continues sont d'ores et déjà programmées pour l'année 2019 à savoir :

- Trois formations de 2 jours concernant la sécurité numérique des systèmes en janvier, mai et juillet,

- Une formation de 10 heures relative aux méthodes de test et testeurs développée pour la société IC'ALPS de Grenoble début mars,

- Une série de séminaires et de formations courtes organisés par CAPTRONIC et réalisés par des enseignants-chercheurs du pôle MIGREST à Strasbourg et Nancy en mars, avril et mai,

- Deux formations d'un jour sur la programmation de carte de prototypage proposées par la société Digilent en mars.

La fin du projet Initiative d'Excellence pour les Formations Innovantes FINMINA au 31 décembre 2019, sera l'occasion pour le GIP CNFM de faire un bilan de ses actions en matière de Formations Continues, d'évaluer les actions mises en place et de se projeter avec un plan d'action pour les années qui viennent.

\section{Remerciements}

Les auteurs souhaitent remercier le GIP-CNFM (1), l'Université de Montpellier (17) et la Région Occitanie (44) pour leur soutien. Ils remercient également tous les directeurs de pôle, sous-directeurs, enseignants-chercheurs et chercheurs qui se sont impliqués dans l'élaboration du catalogue en proposant des fiches de formation, ainsi que les directeurs et chargés de mission des Services de Formation Continue partenaires pour leur confiance et leur collaboration. Enfin, les auteurs remercient l'Agence Nationale de la Recherche (ANR) pour son soutien sous subvention ANR-11-IDFI- $0017 \mathrm{du}$ projet IDEFIFINMINA (5).

\section{Références}

1. GIP-CNFM : Groupement d'Intérêt Public - Coordination Nationale pour la formation en Microélectronique et en nanotechnologies. Site web : http://www.cnfm.fr

2. O. Bonnaud, P. Gentil, A. Bsiesy, S. Retailleau, E. Dufour-Gergam, J.M. Dorkel, GIP-CNFM: a French education network moving from microelectronics to nanotechnologies. Oral communication; in Proc. of EDUCON'11; Amman (Jordan) 3-6 April 2011, ISBN978-1-61284641-5, pp 122-127

3. O. Bonnaud, L. Fesquet, P. Nouet, T. Mohammed-Brahim, FINMINA: a French national project to promote Innovation in Higher Education in Microelectronics and Nanotechnologies, Oral communication, ITHET 2014 York (UK), Proc of ITHET'2014; http: / / www.york.ac.uk / conferences / ithet 2014 /

4. IDEFI-FINMINA : Initiative d'Excellence - Formation Innovante en MIcroélectronique et NAnotechnologies, ANR-11-IDFI-0017. Site web : http: / / www.cnfm.fr/VersionFrancaise / / actualites/FINMINA.htm

5. O. Bonnaud, A. Bsiesy, L. Fesquet, B. Pradarelli, IDEFI-FINMINA: a French educative project for the awareness, innovation and multidisciplinarity in microelectronics, Proc. of EAEEIE'2017 conference, 7-9 June 2017, Grenoble (France)

6. B. Pradarelli, P. Nouet, O. Bonnaud, La formation tout au long de la vie en Microélectronique et en Nanotechnologies dans le cadre du réseau GIP-CNFM , J3eA, Vol 16 1001, 9 pages (2017)

7. Services Nationaux du CNFM : site web : http: / / web-pcm.cnfm.fr

8. Cadence : site web : http: / / www.cadence.com

9. Synopsys : site web : https: / / www.synopsys.com

10. Mentor Graphics : site web : https: / / www.mentor.com 
11. Altera by Intel : https: / / www.altera.com

12. Xilinx : site web : https: / / www.xilinx.com

13. Digilent : site web : http://store.digilentinc.com

14. Plateforme technologique des Services Nationaux du CNFM dédiée à la Sécurité Numérique : site web : http: / / web-pcm.cnfm.fr/secnum/

15. Plateforme technologique des Services Nationaux du CNFM dédiée au test industriel de circuits intégrés : site web : http://web-pcm.cnfm.fr/test/

16. Télécom Paristech : site web : https://www.telecom-paristech.fr/

17. Université de Montpellier : site web : http: / / www.umontpellier.fr

18. GECO : Base de données relative au catalogue de formation continue, hébergée par les Services Nationaux : http://geco.cnfm.fr/

19. Plateforme technologique des Services Nationaux du CNFM dédiée à la Formation Continue : site web : http: / / web-pcm.cnfm.fr / formation /

20. Pôle CNFM PACA : site web : http: / / www.cnfmpaca.fr / cnfmpaca.php

21. Pôle CNFM de Lille : site web : http: / / cnfm.univ-lille1.fr/fr/

22. Service de Formation Continue de l'Université de Strasbourg : site web : https: / / www.unistra.fr

23. Service de la Formation Continue d'Aix-Marseille Université : site web : https://www.univamu.fr/public/formations-courtes-qualifiantes-et-sur-mesure

24. Service de Formation Continue de l'Université de Montpellier: site web: http: / / sfc.edu.umontpellier.fr

25. Service de Formation Continue de l'Université de Lille 1 : site web : http: / / formationcontinue.univ-lille1.fr

26. Service de Formation Continue de l'Université de Paris Sud (Orsay) : site web : http: / / www.upsud.fr / fr / formations / formation-continue.html

27. Service de Formation Continue de l'Université de Nice : site web : http: / / unice.fr / formation / formation-continue

28. Service de Formation Continue de l'INSA de Lyon (INSAVALOR) : site web : http: / / www.insavalor.fr / insavalor / index.html

29. Service de Formation Continue de l'INSA de Toulouse : site web: http://www.insatoulouse.fr/fr/formation/formation_continue.html

30. Service de Formation Continue de Grenoble INP : site web : http: / / formationcontinue.grenoble-inp.fr

31. Compte Facebook du pôle CNFM de Montpellier: https: / / www.facebook.com / CNFMMONTPELLIER /

32. Compte LinkedIn du pôle CNFM de Montpellier : https: / / www.linkedin.com / in / cnfmmontpellier-320888136/

33. Compte Twitter du pôle CNFM de Montpellier : https: / / twitter.com / cnfmmontpellier

34. ACSIEL Alliance Electronique: website: http: / / www.acsiel.fr /

35. Société ST Microelectronics : site web : http: / / www.st.com

36. Société Artcam Production : http://www.artcam-production.com/

37. Chaîne YouTube "CNFM COMMUNICATION" : https: / / www.youtube.com / channel / UC8omjRiW5hXQIUAoT6_g8dg

38. DESU ingénieur produit microélectronique du pôle CNFM PACA Marseille Module: http://geco.cnfm.fr/Formation/fiche/167

39. Electronique Radiofréquence RF : http://geco.cnfm.fr/Formation/fiche/168

40. Protocoles RF (ISO 7816, ISO 14443) et NFC : http://geco.cnfm.fr/Formation/fiche/171

41. Techniques de Fiabilité : http://geco.cnfm.fr/Formation/fiche/170

42. Méthodes statistiques et Bases de données : http://geco.cnfm.fr/Formation/fiche/174

43. Formation continue courte "Sytème d'exploitation temps reel (freertos) dans microcontroleur 32 bits": http://geco.cnfm.fr/Formation/fiche/164 (37)

44. Région Languedoc Roussillon devenue Occitanie : site web : http: / / www.laregion.fr 